OPEN ACCESS

Edited by:

Joshua Michael Bauml,

University of Pennsylvania,

United States

Reviewed by:

Sandip Patel,

University of California, San Diego,

United States

Giulio Metro,

Hospital of Santa Maria della

Misericordia in Perugia, Italy

*Correspondence:

Jarushka Naidoo

jnaidoo1@jhmi.edu

Specialty section:

This article was submitted to

Thoracic Oncology,

a section of the journal

Frontiers in Oncology

Received: 02 April 2019

Accepted: 31 May 2019

Published: 20 June 2019

Citation:

Connolly C, Bambhania K and Naidoo J (2019) Immune-Related Adverse Events: A Case-Based Approach. Front. Oncol. 9:530 doi: 10.3389/fonc.2019.00530

\section{Immune-Related Adverse Events: A Case-Based Approach}

\author{
Caoilfhionn Connolly ${ }^{1}$, Kalindi Bambhania ${ }^{2}$ and Jarushka Naidoo ${ }^{2,3 *}$ \\ ${ }^{1}$ Department of Internal Medicine, Johns Hopkins Hospital, Baltimore, MD, United States, ${ }^{2}$ Department of Oncology, Sidney \\ Kimmel Comprehensive Cancer Center at Johns Hopkins University, Baltimore, MD, United States, ${ }^{3}$ Department of \\ Oncology, Bloomberg-Kimmel Institute for Cancer Immunotherapy, Baltimore, MD, United States
}

Immunotherapy has heralded the advent of a new era in oncology. Immune checkpoint inhibitors (ICls) enhance anti-tumor immunity, thereby reinvigorating a patient's immune system to fight cancer. While therapy with this class of agents has resulted in improved clinical outcomes for patients with multiple tumor types, a broad spectrum of immune-related adverse events (irAEs) may affect any organ system, with variable clinical presentations. Prompt recognition and management of irAEs are associated with improved irAE outcomes, and represents an important new clinical challenge for practicing oncologists. Herein, we provide a comprehensive case-based review of the most common and clinically-important irAEs, focussing on epidemiology, clinical manifestations, and management. We also examine future strategies that may provide meaningful insights into the prevention and management of irAEs.

Keywords: immune checkpoint inhibitors, immune-related adverse events, immunotherapy, immune-related toxicities, management

\section{INTRODUCTION}

Immune evasion is one of the hallmarks of cancer cells (1). Immune checkpoints are negative regulators of immune activation; exploiting the action of checkpoints such as cytotoxic Tlymphocyte antigen 4 (CTLA-4), programmed cell death (PD-1), and its ligand programmed cell death ligand 1 (PDL-1) allows cancer cells to evade immune-surveillance, thus enabling unchecked tumor growth. Immune checkpoint inhibitors (ICIs) negate this key mechanism of cancer progression, revitalizing the immune system to eradicate cancer cells. However, unleashing the effects of the immune system in this manner can result in a unique spectrum of toxicities known as immune-related adverse events (irAEs). ICIs have demonstrated unprecedented response rates in a wide array of cancer types, with seven checkpoint inhibitors currently approved by the FDA and in over 14 different cancer treatment indications (Table 1). As ICIs enter routine clinical practice, clinicians across all sub-specialties are increasingly faced with the diagnostic and therapeutic challenge of irAE identification and management.

The incidence of irAEs has been reported to range from 15 to $90 \%$ in late phase clinical studies $(2,3)$. The risk, clinical manifestations, and severity of irAEs is variable across ICI regimen and cancer type. The frequency and severity of irAEs appears greatest amongst patients receiving ipilimumab/nivolumab combination therapy compared to monotherapy (4-6). Patients receiving CTLA-4 therapy more commonly present with colitis and hypophysitis, while patients receiving PD-1 therapy more commonly present with pneumonitis and thyroiditis (7-9).

It is widely recognized that effective management of irAE is dependent on early recognition and prompt intervention (10). High clinical suspicion, timely evaluation and multi-disciplinary management provide the foundation for optimal clinical outcomes (Figure 1). 
The severity of adverse events can be graded as per all adverse events, utilizing the Common Terminology Criteria for Adverse Events (CTCAE) (Table 2) (11). While this grading system has been used in oncology for many years, this is not specifically tailored toward irAEs, and should be supplemented by clinical judgement by those familiar with irAEs. In response to the clinical need to recognize and manage this class of toxicities, comprehensive guidelines to facilitate appropriate evaluation and management of irAEs were recently published by key oncology societies, including the American Society of Clinical Oncology (ASCO), the European Society for Medical Oncology (ESMO), the Society for Immunotherapy of Cancer Toxicity Management Working Group and the National Comprehensive Cancer Network (11-13). These guidelines are largely based on retrospective data and expert opinion. While they detail commonly encountered irAEs, rarer toxicities are not universally featured, and there is a paucity of guidance regarding severe or refractory irAEs, or those in which little has been published. Importantly, a recent meta-analysis demonstrated that fulminant irAEs resulting in patient death have been reported at a rate of $0.3-1.3 \%$ (14). Fulminant presentations are particularly challenging, and in the absence of prospective clinical data, management remains variable. While management varies according to severity of encountered irAE, ICI therapy can be continued for most grade 1 toxicities, with suspension of ICI for grade 2 or greater toxicities. Resumption of therapy following resolution of symptoms is a frequent clinical scenario that is currently lacking data. Retreatment can be considered for grade 2 or 3 toxicities when symptoms revert to grade 1 or less, however, permanent discontinuation of therapy is recommended with grade 4 irAEs $(10,12,13)$. In a recent study of patients with NSCLC, retreatment with anti-PD-L1 therapy resulted in recurrence of irAEs in 52\% of patients (15). The safety and benefit of retreatment is unknown and the decision to retreat should be considered on a case-by case basis.

This review provides a practical guide to what is known about irAEs and how to optimize evaluation and management of irAE utilizing a case-based approach. Within common or rare but clinically-important cases, we highlight the current stateof the art in terms of diagnosis and management, as well as emerging challenges. Finally, we discuss new therapies and novel approaches that may alleviate the future burden of irAE.

\section{ORGAN-SPECIFIC IRAES}

Given the wide array of clinical manifestations of irAEs, we summarize the diagnosis, management and emerging data in the most common or clinically-challenging irAEs that clinicians may encounter by organ system. Figure 2 illustrates some of the irAE manifestations encountered at the Johns Hopkins Hospital.

\section{RHEUMATIC}

\section{Case 1: Inflammatory Arthritis}

Clinical Presentation: A 58-year old woman is treated with combination Ipilimumab/Nivolumab therapy for stage IV non-small-cell lung carcinoma (NSCLC), and presents with a 3-week history of a left, swollen, and painful left knee.
TABLE 1 | FDA-approved ICls and their indications.

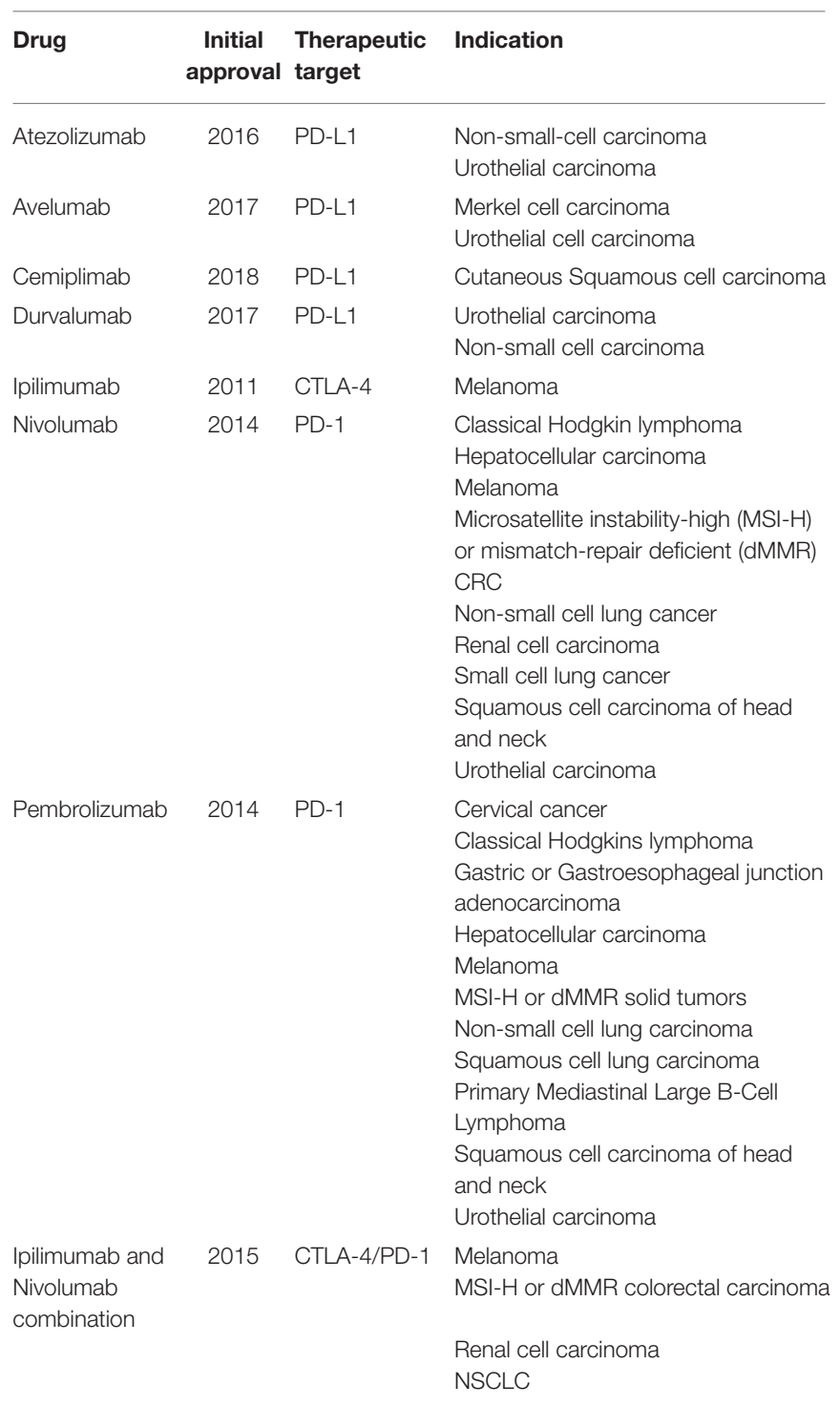

PD-1, programmed cell death protein; PD-L1, programmed cell death ligand-1; CTLA-4, cytotoxic Tlymphocyte-associated antigen 4.

Arthralgia and inflammatory arthritis (IA) are the most commonly encountered rheumatic irAEs $(16,17)$. ICI-induced IA can have a variable timing, with a median onset 5 months (range 1-24 months) after ICI initiation (18). The clinical presentations of IA are variable with oligoarthritis, polyarthritis, and reactive arthritis-like manifestations being described (19). Cappelli et al. reported that patients who received combination ICIs were more likely to present with large joint involvement and to already have another irAE, while patients treated with ICI monotherapy were more likely to have initial small joint involvement and to have IA as their only irAE (16). Other rheumatic irAEs include polymyalgia-like syndrome, vasculitis, sicca syndrome, and inflammatory myopathies (20). The data regarding the incidence of rheumatic complications is highly variable, with rates of arthralgia ranging from 1 to $43 \%$, with reports of other manifestations ranging from 0.7 to 


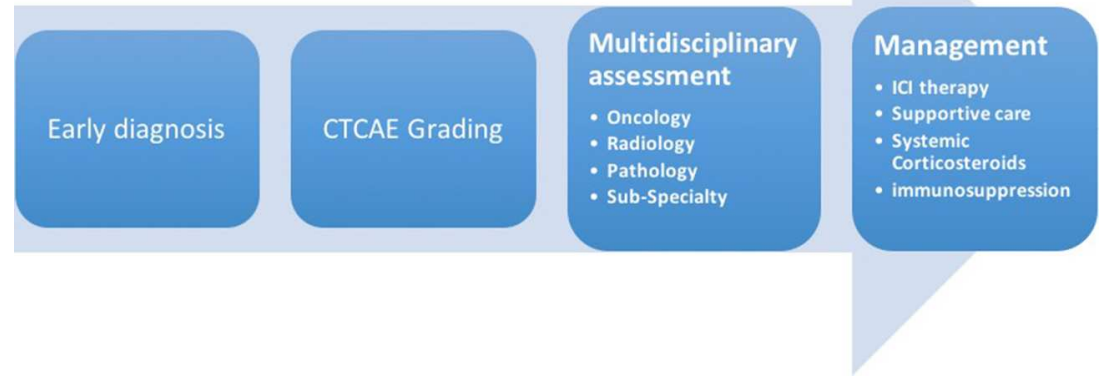

FIGURE 1 | Early diagnosis and multidisciplinary management are integral to optimal clinical outcomes. It is crucial that physicians have a detailed knowledge of the wide range of clinical manifestations of irAEs, as well as familiarity with existing algorithms for their grading and management.

TABLE 2 | Common terminology criteria for adverse events grading.

\begin{tabular}{|c|c|c|c|}
\hline CTCAE grade & Level of care & Management & ICI therapy \\
\hline 1: Asymptomatic or Mild & Ambulatory & Observation & $\begin{array}{l}\text { Continue } \mathrm{ICl} \text { therapy with close } \\
\text { monitoring }\end{array}$ \\
\hline 2: Moderate & Ambulatory & $\begin{array}{l}\text { Systemic corticosteroids }(0.5-1 \mathrm{mg} / \mathrm{kg} / \text { day of prednisone or } \\
\text { equivalent) }\end{array}$ & $\begin{array}{l}\text { Temporarily hold; resume when grade } \\
1 \text { or less }\end{array}$ \\
\hline $\begin{array}{l}\text { 3: Severe but not } \\
\text { immediately life-threatening }\end{array}$ & Inpatient & $\begin{array}{l}\text { High dose systemic corticosteroids }(1-2 \mathrm{mg} / \mathrm{kg} / \mathrm{d} \text { prednisone } \\
\text { or methylprednisolone); consider additional therapies if no } \\
\text { response with } 48-72 \mathrm{~h}\end{array}$ & $\begin{array}{l}\text { Temporarily hold; resume when grade } \\
1 \text { or less in discussion with patient }\end{array}$ \\
\hline 4: Life-threatening & $\begin{array}{l}\text { Inpatient }+/- \text { intensive } \\
\text { care unit }\end{array}$ & $\begin{array}{l}\text { High-dose corticosteroids ( } 1-2 \mathrm{mg} / \mathrm{kg} / \mathrm{d} \text { prednisone or } \\
\text { methylprednisolone); consider additional therapies if no } \\
\text { response with } 48-72 \mathrm{~h}\end{array}$ & $\begin{array}{l}\text { Permanent discontinuation with the } \\
\text { exception of endocrinopathies } \\
\text { managed by hormone replacement }\end{array}$ \\
\hline
\end{tabular}

$5.1 \%(21,22)$. This is potentially related to the variability in potential coding of these events in clinical trials, using CTCAE criteria.

\section{Diagnostic Evaluation}

Patients should undergo a full musculoskeletal evaluation. Laboratory studies including ESR (Erythrocyte sediment rate), CRP (C-reactive protein), RF (rheumatoid factor), ACPA (anticitrullinated peptide/protein antibodies), ANA (anti-nuclear antibody), and HLA-B27 (Human Leukocyte Antigen B-27) should be sent, to help differentiate between phenotypes of IA that may have treatment implications. The majority of patients are seronegative, but a seropositive subgroup has been described (16). Imaging including joint ultrasound or MRI should be completed to assess for effusion, erosive disease and tenosynovitis. In the above patient, diagnostic evaluation was notable for left knee effusion (see Figure 2A) with unremarkable synovial fluid analysis, elevated inflammatory markers, and seronegative disease.

\section{Management}

Early recognition of IA is critical to avoid erosive joint damage. ICI can be continued for grade 1 toxicities and treated with simple analgesia consisting of acetaminophen and non-steroidal anti-inflammatory drugs (NSAIDs). However, in this patient with grade 2 IA, ICI was temporarily held while she received treatment with acetaminophen, NSAIDs and oral corticosteroids of prednisone $20 \mathrm{mg} /$ day for 4 weeks. Given large joint involvement, she also received an intra-articular corticosteroid injection. Corticosteroid dose can be adjusted pending clinical response with taper over 4-6 weeks in the instance of clinical improvement, or, in presence of progressive IA, treatment can be escalated with addition of other immunomodulatory medications such synthetic or biologic disease-modifying anti rheumatic drugs (DMARDs). This patient demonstrated improvement and prednisone was tapered over 4 weeks. Ipilimumab/Nivolumab therapy was resumed when IA symptom control was reached, and the patient was taking a prednisone dose of $10 \mathrm{mg}$ daily. A notable feature of rheumatic irAEs, in particular ICI-inducedIA, is their predilection for persistence despite cessation of ICI therapy which may require long-term immunomodulatory therapy for months to years after diagnosis (23). Due to likely prolonged treatment requirements, physicians should consider starting immunomodulatory medications earlier than one would with other irAEs. DMARDs should be considered in all patients with grade 3 or 4 disease, as well as grade 2 patients that display progression of disease with corticosteroid therapy. In steroid-refractory cases, a common approach is to start with Methotrexate, with escalation to biologic agents such as infliximab in the absence of adequate clinical response. 

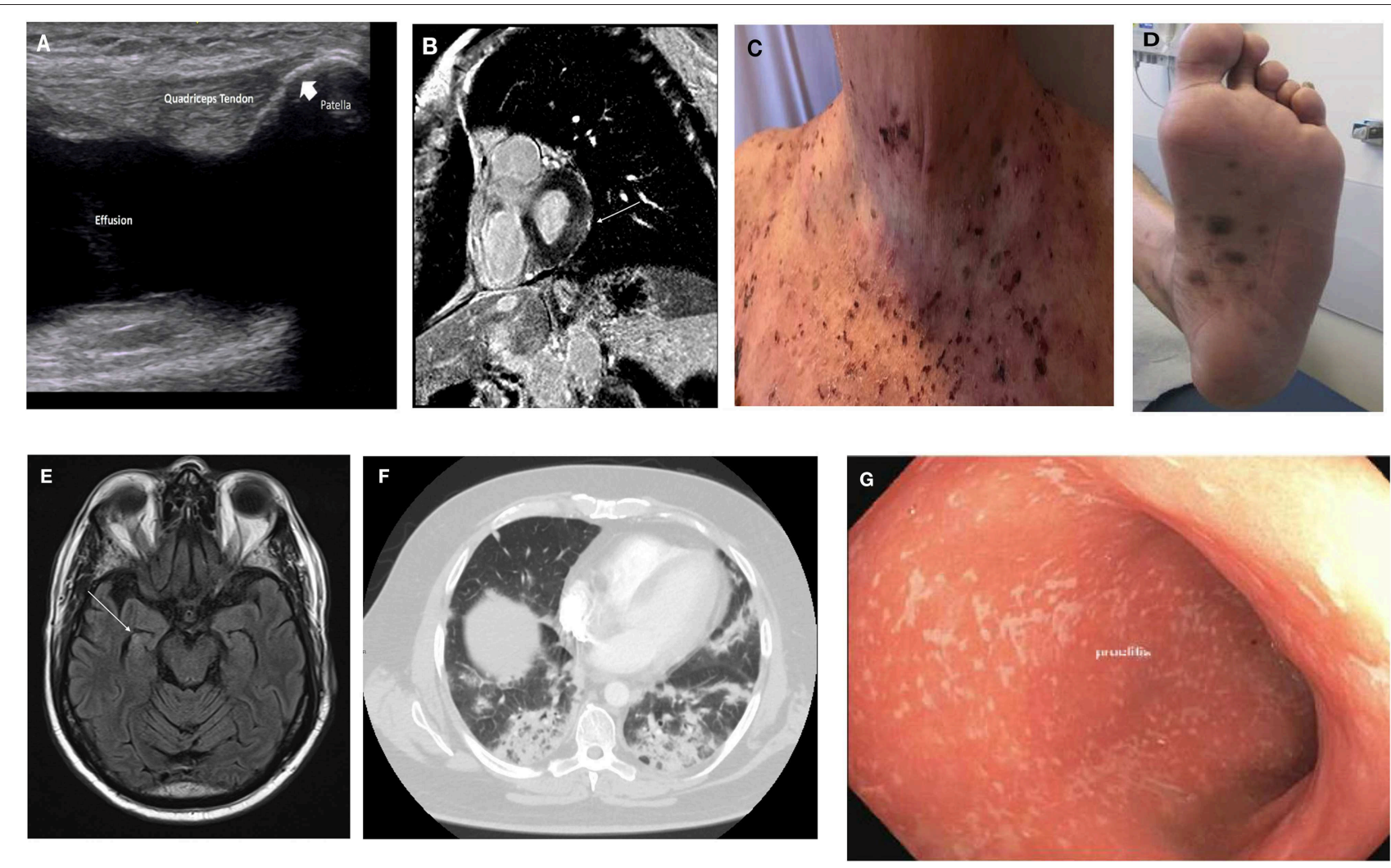

FIGURE 2 | Clinical manifestations of irAE. (A) Inflammatory arthritis: US image of knee effusion; (B) myopericarditis: cardiac MRI with late gadolinium enhancement overlying basal left ventricular lateral wall (arrow); (C) bullous pemphigoid; (D) lichenoid dermatitis; (E) encephalitis: brain MRI with hyperenhancement of right hippocampus (arrow); (F) pneumonitis: chest CT with bilateral lower lobe infiltrates; (G) colitis: endoscopic findings of pan-colitis.

\section{Case 2: Temporal Arteritis}

Clinical Presentation: A 76-year old man with advanced urothelial presents with temporal headache and jaw claudication 10 days after cycle two of durvalumab.

Both polymyalgia-like syndrome and giant cell arteritis (GCA) have been reported following treatment with ICI. A recent analysis of WHO's VigiBase found that patients who received ICI had a reporting odds ratio of GCA 13 times greater than patients not treated with ICI (24). This study also reported that the median time of onset from last dose of ICI was 55 days (range: 21-98) with a greater predilection for elderly patients, Caucasian patients, and those with melanoma (24). ICI-induced GCA symptoms mirror those of traditional GCA, including temporal headache, jaw claudication, monocular vision loss, unexplained fever, and fatigue.

\section{Diagnostic Evaluation}

Early diagnosis is vital to prevent devastating ocular and cerebrovascular complications of GCA. Visual impairment has been reported in $28 \%$ of patients with ICI-induced GCA (24). The diagnosis of GCA should not be made based upon symptoms alone and investigations including complete blood count (CBC), ESR, and CRP. Temporal artery biopsy is the gold standard diagnostic test and provides definitive diagnosis, but should not delay treatment. In this patient, physical examination was notable for temporal artery tenderness with intact vision. Initial investigations were notable for markedly elevated ESR and CRP.

\section{Management}

Given intact vision, the patient was commenced on prednisone $60 \mathrm{mg} /$ day to complete 2 weeks of therapy followed by a taper every 2 weeks. Durvalumab was held pending clinical response. Temporal artery biopsy confirmed the diagnosis. This management was instituted with the input of a rheumatology consult.

Patients with suspected ICI-induced GCA should be managed as per traditional rheumatic GCA with the addition that ICI therapy should be held pending clinical improvement in GCA. In patients without visual loss at diagnosis, treatment should comprise prednisone $1 \mathrm{mg} / \mathrm{kg} /$ day (maximum dose of $60 \mathrm{mg}$ daily). Patients with threatened or established visual loss at diagnosis should be commenced on intravenous pulse corticosteroids ( $1 \mathrm{~g}$ methylprednisolone daily) for 3 days followed by high dose oral therapy (25). Oral corticosteroids should be maintained for 2-4 weeks followed by a two-weekly interval taper. Typically, prednisone will be tapered by $10 \mathrm{mg}$ every 2 weeks until a dose of $40 \mathrm{mg} /$ day is reached, at which point the 
dose will be reduced by $5 \mathrm{mg}$ decrements every 2 weeks until a dose of $20 \mathrm{mg} /$ day is reached. At this point, the rate of the corticosteroid taper is slowed.

In the case of relapsed GCA symptoms despite high-dose corticosteroids, abatacept has demonstrated efficacy (26), while methotrexate and tocilizumab are alternate therapeutic options. Resumption of ICI therapy can be considered once prednisone dose is $<10 \mathrm{mg} /$ day, ideally in consultation with rheumatology.

\section{CARDIOVASCULAR}

\section{Case 1: Myocarditis}

Clinical presentation: A 65-year old man with advanced renal cell carcinoma presents with chest pain and dyspnea following 1 cycle combination Ipilimumab/Nivolumab therapy.

Myocarditis is the most commonly documented cardiac irAE (27). Cardiovascular complications of ICIs are less wellrecognized, but these complications can be potentially fatal $(24,28)$. The absolute incidence of cardiac irAE is reported at $<1 \%$, however the true incidence is likely higher given prior under-recognition of cardiac toxicity (26). Recently, Salem et al. reported that the odds of myocarditis in patients receiving ICIs was 11 times greater than patients who did not receive ICI (24), with a median time of onset 30 days after initial exposure to therapy. Wang et al. recently found that myocarditis has the highest fatality rate of any irAE (14). Manifestations of myocarditis are variable, with a clinical spectrum ranging from fatigue, chest pain, acute heart failure to cardiogenic shock, arrhythmias, and sudden death $(29,30)$. Pericarditis, conduction disease and ventricular arrhythmias are other reported cardiac irAEs, but acute myocardial ischemia, new onset systolic dysfunction and Takotsubo syndrome can also occur (31).

\section{Diagnostic Evaluation}

Myocarditis is characterized by elevated cardiac enzymes (troponin, pro-BNP), with/without the onset of left ventricular dysfunction and evidence of myocardial inflammation on cardiac MRI or PET/CT; all of these parameters should be investigated. In cases of uncertainty, endomyocardial biopsy can be useful although non-invasive investigations are preferred. Initial evaluation of this patient was notable for marked hypervolemia and pulmonary edema. Diagnostic workup was notable for non-specific ST-segment changes on ECG, marked elevation of cardiac markers and new reduced ejection fraction on transthoracic echocardiogram. Cardiac MRI was notable for late gadolinium enhancement overlying the basal left ventricular lateral wall (see Figure 2B).

\section{Management}

The patient was transferred to the Cardiac Care Unit under the care of the cardiology team. ICI was permanently discontinued. The patient was monitored on continuous telemetry and commenced treatment with daily intravenous $1 \mathrm{~g}$ methylprednisolone and diuretic therapy. His hospital course was complicated by complete heart block that was managed with transvenous pacing, however, progressive clinical deterioration followed, resulting in cardiac arrest from which he could not be resuscitated.
All grades of cardiac toxicity warrant evaluation given the risk of cardiac compromise. Effective management requires close monitoring with a multimodal therapeutic plan consisting of ICI cessation, high-dose corticosteroids (1-2 mg/kg of prednisone/day or equivalent) and early cardiology consultation with management of cardiac complications. Steroid-refractory cases may necessitate the addition of mycophenolate, infliximab, or anti-thymocyte globulin, and there are no specific data that demonstrate a superior approach of these three options. Conduction disease is emerging as a common and potentially serious cause of ICI-medicated sudden death, even in the absence of myocarditis (32). Electrophysiology consultation should be completed for consideration of insertion of cardiac device (pacemaker or defibrillator) if there is concern for ICIinduced conduction disease. In the instance of cardiac device insertion, the decision to proceed with ICI therapy should be made in conjunction with the patient, cardiology, and oncology. A surveillance strategy has recently been proposed that suggests a baseline cardiac assessment for all patients including baseline cardiac risk factor assessment, electrocardiogram, cardiac troponin, and pro-brain natriuretic peptide (pBNP) in addition to a non-invasive surveillance protocol for patients with cardiac risk factors defined as pre-existing coronary artery disease, hypertension, diabetes mellitus, obesity, smoking history, or positive family that should be completed within the initial 12 weeks of therapy (32). Given the mortality risk of these complications this approach may be appropriate, but should be evaluated in prospective studies.

\section{DERMATOLOGIC}

\section{Case 1: Maculopapular Rash}

Clinical presentation: A 61-year old woman with Merkel cell carcinoma presents with a pruritic rash after cycle two of Avelumab therapy. She denies mouth pain, eye pain, fever, or constitutional symptoms.

Cutaneous toxicities are the most commonly encountered irAE, and has been reported in $30-50 \%$ of patients receiving ICI therapy (33), with $37-70 \%$ of patients receiving CTLA- 4 and $17-37 \%$ of patients receiving PD1/PDL-1 therapy experiencing dermatologic toxicities, respectively $(2,3)$. Of these, $<3 \%$ experience greater than grade 3 toxicity. Dermatologic irAEs are challenging as they have variable clinical presentation and timing of onset. Clinical manifestations range from pruritus, vitiligo, inflammatory rashes (maculopapular eruption, dermal hypersensitivity reactions, acneiform, exfoliative, and psoriasiform lesions), bullous dermatoses (bullous pemphigoid, bullous drug reaction) to severe cutaneous adverse reactions (Stevens Johnson Syndrome, Toxic Epidermal Necrolysis, Drug-induced hypersensitivity syndrome/Drug reaction with eosinophilia and systemic symptoms). Time to onset can vary between 2 weeks and several months from onset of therapy $(34,35)$.

\section{Diagnostic Evaluation}

In patients with cutaneous irAE, a thorough clinical history and physical examination should be obtained. Clinicians should perform a close evaluation of all skin surfaces, mucus 
membranes, and lymph nodes with a specific focus on the percentage body surface area that is involved and the presence or absence of blistering. A positive Nikolsky sign (induction of blistering via mechanical pressure) should prompt concern for severe cutaneous reaction (SJS/TEN), which will characteristically include mucosal and systemic involvement (fever, constitutional symptoms). On examination, this patient was noted to have tense blisters and erosions on her extremity flexures that involved $15 \%$ body surface area. Nikolsky sign was positive. There was no evidence of ocular of mucosal involvement. Hematological and biochemical investigations were normal. She was evaluated by a dermatologist and underwent lesional and perilesional biopsies that confirmed the diagnosis of bullous pemphigoid. Skin biopsy revealed linear immunoglobulin G (IgG) and linear C3 staining along the basement membrane zone, which is present in $>90 \%$ of cases (36). If biopsy is not possible, serum can be sent for antibodies to BP180 and BP230 (ELISA testing) to confirm the diagnosis (36).

\section{Management}

The patient was treated with betamethasone topical therapy and oral prednisone $1 \mathrm{mg} / \mathrm{kg}$, tapered over 4 weeks. The patient was monitored closely with serial photography for progression but displayed evidence of improvement. Avelumab was initially held, but resumed upon resolution of symptoms to less than grade 1 severity.

With the exception of bullous disease, most grade 1 and 2 cutaneous toxicities can be managed with topical therapies (emollients, corticosteroids) and continuation of immunotherapy. Escalation of dermatologic care includes holding immunotherapy, increasing the potency of topical corticosteroids and starting systemic corticosteroids. Grade 4 toxicities should be treated with intravenous methylprednisolone dosed at 1-2 mg/kg. Patients with $>30 \%$ body surface area involvement should be managed in specialist burns unit. In steroid-refractory cases, IVIG or cyclosporine can be considered in conjunction with dermatology. Notably, cutaneous irAEs have been recognized as a barrier to ICI compliance (11). Interestingly, development of cutaneous toxicities may correlate with clinical response in patients with metastatic melanoma, with a greater survival benefit reported in melanoma patients who developed rash or vitiligo after pembrolizumab or nivolumab therapy, respectively $(37,38)$. Development of new vitiligo has also demonstrated a significant association with both progressionfree survival and overall survival in a meta-analysis of 27 studies of melanoma patients treated with a wide variety of immunotherapeutic strategies (139 treatment arms comprising 11 general immune stimulation, 84 vaccine, 28 antibody-based, and 16 adoptive transfer)(39).

\section{RENAL}

\section{Case 1: Nephritis}

Clinical presentation: A 58-year old woman with stage IV PD-L1+ NSCLC was noted to have asymptomatic creatinine (Cr) elevation to $2.5 \mathrm{mg} / \mathrm{dl}$ (baseline $0.9 \mathrm{mg} / \mathrm{dl}$ ) after 2 cycles of Pembrolizumab.
Nephritis is the most common renal toxicity of anti-PD-1/PDL1 therapy, and is more common in patients with NSCLC treated with the combination of chemotherapy and immunotherapy, which is now standard first-line therapy for patients with advanced NSCLC (40). Hyponatremia may also be encountered in these cases, however this occurs more commonly in the setting of hypophysitis (41). There is significant heterogeneity in the onset of kidney injury; with CTLA-4 nephrotoxicity occurring earlier (range: 2-3 months), compared to the later onset of injury with PD-1 related nephrotoxicity (range: 3-10 months) (4244). Acute interstitial nephritis is the most prevalent pathologic lesion, with one report of thrombotic microangiography (41). While initial data suggest that ICI-mediated renal injury ranged from 1 to $2 \%$ in monotherapy and $4.5 \%$ in combination therapy (45), more recent studies have suggested a higher incidence ranging from 9.9 to $29 \%$ (46).

\section{Diagnostic Evaluation}

Patients with renal irAEs are frequently asymptomatic and therefore, routine monitoring of renal indices (serum creatinine, electrolytes) is necessary to ensure prompt detection. Symptomatic patients may present with nausea, vomiting, fatigue, altered mental status, reduced urinary output, peripheral edema, or dyspnea. All patients should undergo complete renal evaluation including urinalysis, serum creatinine, serum electrolytes, and consideration for renal ultrasound to evaluate for other potential etiologies. In this asymptomatic patient with $\mathrm{Cr} 2.5 \mathrm{mg} / \mathrm{dl}$, urinalysis was notable for pyuria with mild peripheral eosinophilia on CBC. Investigations may reveal pyuria (68\%), hematuria (16\%), and/or proteinuria on urinalysis with eosinophilia $(21 \%)$ on CBC $(41,42)$.

\section{Management}

Therapy should be temporarily withheld while evaluation for an underlying cause of nephrotoxicity is completed. Should no alternate cause be identified, patients should be presumed to have immune-mediated toxicity. Reflex kidney biopsy is not recommended until corticosteroid treatment has been attempted. Corticosteroids are the mainstay of treatment. Additional immunosuppression including mycophenolate can be considered in steroid-refractory cases. In this patient, Pembrolizumab was held temporarily. Nephrology were consulted and she was commenced on prednisone 1 $\mathrm{mg} / \mathrm{kg} /$ day. At 2-week follow up Cr had improved to $1.8 \mathrm{mg} / \mathrm{dl}$. Prednisone was tapered over 4 weeks with improvement in renal indices to baseline. Following discussion with the patient regarding risks and benefits, pembrolizumab was resumed.

\section{NEUROLOGY}

\section{Case 1: Encephalitis}

Clinical presentation: A 62-year old female with stage IV renal cell carcinoma presents with headache and altered mental status 1 week following Ipilimumab/Nivolumab therapy. 
Neurological toxicities, while uncommon, are of special interest due to their potential severity. These complications encompass dysregulation of both central and peripheral nervous systems. Central manifestations include encephalitis, aseptic meningitis, transverse myelitis and posterior reversible encephalopathy syndrome (PRES). Encephalitis is estimated to occur in $0.1-0.2 \%$ of patients (47). Patients may present with headache, altered mental status, motor or sensory deficits, abnormal behaviors, personality change, speech disorders, or movement disorders. A meta-analysis of 9,208 patients who received ICI therapy reported that incidence of neurologic irAEs ranged from 3.8 to $6.1 \%$ with anti-CTLA4 and anti-PD-1 monotherapy, respectively, and up to $12.0 \%$ with combination therapy. High-grade events were reported at an incidence of $<1 \%$ across all ICIs (47). The median time of onset to encephalitis is 6 weeks. Most neurological irAEs present initially with non-specific symptoms such as headache, dysgeusia and sensory impairment $(47,48)$.

\section{Diagnostic Evaluation}

A baseline complete blood count, liver, renal, and thyroid function should be sent as well as assessment of the pituitary axis. If there is concern for a vasculitic process, ESR, CRP, and ANCA should be sent. Lumbar puncture should be completed to evaluate for infection and leptomeningeal disease. Anti-N-methyl-Daspartate receptor (anti-NMDAR) antibody should also be sent. Contrast-enhanced MRI brain scan and EEG monitoring can be completed to assesses for vascular insult, brain metastasis and subclinical seizure activity. In this patient, the diagnostic evaluation revealed an intact pituitary axis, with a subtle hyperintensity of the right hippocampus on MRI (see Figure 2E). CSF analysis was notable for a lymphocytic pleocytosis with negative infectious, paraneoplastic and cytopathology panel. EEG showed diffuse non-specific slowing.

\section{Management}

Prompt recognition and expeditious management carries the potential of complete neurological recovery $(48,49)$. In this patient, ICI was held and she was commenced on IV acyclovir that was discontinued on receipt of negative viral PCR panel. Neurology were consulted. She was treated with pulse corticosteroids (methylprednisolone $1 \mathrm{~g}$ IV daily for 5 days) as well as IVIG $2 \mathrm{~g} / \mathrm{kg}$ over 5 days. Following 8 days of therapy with corticosteroids and IVIG, she demonstrated full neurological recovery. A recent case series of nine patients with ICI-induced neurological toxicity reported that $77.8 \%$ of patients showed marked symptomatic improvement following discontinuation of immunotherapy and management with corticosteroids (49). In steroid-refectory cases, additional therapeutic modalities such as IVIG, rituximab or plasmapheresis can be considered, since this toxicity is autoantibody-mediated.

\section{Case 2: Myasthenia Gravis}

Clinical Presentation: A 67-year old man with metastatic colorectal cancer presents with diplopia 1 week following the first cycle of Nivolumab therapy.
Myasthenia gravis is estimated to occur in $0.1-0.2 \%$ of patients receiving immunotherapy $(50,51)$. Presentation is typically within 2-3 weeks of treatment initiation $(52,53)$ with symptoms of fluctuating motor weakness and fatigue that is often associated with ocular and bulbar dysfunction. Peripheral neurotoxicity can also manifest as peripheral neuropathy, autonomic neuropathy, Guillain-Barre syndrome, and necrotizing myositis.

\section{Diagnostic Evaluation}

Any concern for myasthenia gravis warrants rapid evaluation and intervention given the potential for respiratory compromise. In this patient, acetylcholine receptor antibodies were positive. Serial pulmonary function test with negative inspiratory force (NIF) and vital capacity did not reveal respiratory compromise. Electrophysiologic testing (Single fiber EMG) confirmed the diagnosis of myasthenia gravis. Other investigations including creatinine kinase, aldolase, ESR, and CRP should be sent to assess for concurrent myositis. High clinical suspicion for concurrent myositis and myocarditis is warranted given possibility of coexisting myasthenia gravis, myositis and myocarditis in a subset of patients as evidenced in $25 \%$ of cases of nivolumabrelated myasthenia gravis (52).

\section{Management}

ICI-induced myasthenia gravis has been associated with a higher incidence of myasthenic crisis than idiopathic myasthenia gravis $(52,53)$. Thus, a high level of suspicion and rapid initiation of corticosteroids are mandatory to prevent clinical deterioration, which can result in respiratory failure and death. In this patient, nivolumab therapy was held. Neurology were consulted and he was commenced on pyridostigimine $30 \mathrm{mg}$ four times per day as well as prednisone $1 \mathrm{mg} / \mathrm{kg} /$ day. With clinical improvement, prednisone was slowly weaned in $5 \mathrm{mg}$ decrements every 2 weeks. Patients with grade 3 or 4 toxicity should be monitored in the intensive care setting given risk of respiratory compromise. Pyridostigimine can be titrated to achieve optimal relief of symptoms. Additional therapeutic modalities include IVIG or plasmapheresis that should be initiaited for grade 3 or 4 disease.

\section{OCULAR}

\section{Case 1: Uveitus}

Clinical Presentation: A 71-year old man with history of urothelial cancer noted onset of right eye pain 5 weeks following cycle 1 of Atezolizumab therapy.

Ophthalmic irAEs affect $<1 \%$ of ICI-treated patients and typically manifest as uveitis and/or dry eye $(54,55)$. Ocular irAE have a median onset of 2 months and are more commonly associated with other concurrent irAEs (55). Patients can present with eye pain, erythema, pain with eye movement, visual disturbance, diplopia, or photophobia. Less commonly encountered ocular manifestations include inflammatory orbitopathy, keratitis, choroidal neovascularization, serous retinal detachment, retinopathy, neuroretinitis, and ocular myasthenia gravis. 


\section{Diagnostic Evaluation}

Ocular irAEs are commonly seen in associated with other systemic irAEs, therefore clinical suspicion for other manifestations should be high. This particular patient endorsed the presence of visual floaters, but denied pain with eye movement, change in color perception, visual change, or photophobia. On examination, the patient's right pupil was mildly constricted, reactive to light with erythema of the limbus. Left pupil was round and reactive. Color vision and visual acuity were intact. Red reflex was present bilaterally. There was no evidence of concurrent irAE.

\section{Management}

The patient was prescribed topical corticosteroid, 1\% cyclopentolate (topical cyclopegic agent) and prednisone $60 \mathrm{mg}$ daily. An urgent ophthalmology appointment was scheduled within a week. Atezolizumab was temporarily held until completion of corticosteroid taper over 2 months.

The majority of ocular irAE do not necessitate discontinuation of ICI and are managed with topical therapies. All patients should be referred to ophthalmology for slit-lamp and dilated fundus examination to assess for presence of leukocytes in the anterior chamber of the eye as well as to examine the extent of inflammation. In this case, ICI was temporarily held in the setting of grade 2 toxicity. However, ICI should be permanently discontinued with emergent ophthalmology assessment in higher grade irAEs. Additional therapeutic strategies include systemic and topical/intravitreal corticosteroids. Infliximab can be considered in steroid refractory cases (56).

\section{PULMONARY}

\section{Case 1: Pneumonitis}

Clinical presentation: A 54-year-old man with advanced urothelial carcinoma presents following the fifth dose of Durvalumab with dyspnea.

Checkpoint-inhibitor pneumonitis (CIP) is defined as the development of new infiltrates on chest imaging with dyspnea or other respiratory symptoms in the absence of infection, cardiac dysfunction or tumor progression. Presentations can be heterogeneous, ranging from asymptomatic radiographic findings, chest pain, cough, or dyspnea, to life-threatening respiratory compromise (57). The overall incidence of CIP ranges from 0 to $10 \%$, with a median time to onset of $\sim 3$ months reported by Naidoo et al. (8). Patients receiving combination ICI therapy are at increased risk of CIP (10 vs. $3 \%$, respectively; $p$ $<0.001$ ), with evidence to suggest that these patients experience symptoms earlier in the clinical course $(8,57)$. In a study of fatal ICI-associated toxic effects, anti-PD-1/PD-L1-related fatalities were often from pneumonitis, consisting of $35 \%$ of all fatalities (14). The data would suggest that higher grade CIP tend to occur within the first 100-200 days of therapy initiation (57). Emerging data from the Johns Hopkins Hospital group has shown that tumor histology may be a risk factor for CIP in NSCLC patients (58). Furthermore, multistate modeling has demonstrated that NSCLC patients who develop CIP may have a poorer survival (59).

\section{Diagnostic Evaluation}

The patient underwent thorough history and physical examination that was notable only for hypoxia with $\mathrm{O}_{2}$ saturation of $88 \%$ on room air. Physical examination in CIP can be very unrevealing and thus clinicians must be vigilant for early detection. The differential diagnosis should include respiratory infection, rare respiratory infections such as PCP or aspergillosis (especially if being treated with highdose corticosteroids), tumor progression, radiation-induced pneumonitis, and ICI-induced myocarditis/cardiac failure. Further diagnostic evaluation should include infectious evaluation (urine, respiratory culture, viral culture/swab, blood cultures, serum galactomannan), CT imaging and consideration for bronchoscopy with bronchoalveolar lavage (BAL) +/- lung biopsy. This patient underwent CT imaging with findings as illustrated in Figure 2F. Infectious evaluation was negative for the presence of an infectious organism. Bronchoscopy with BAL was notable for the presence of chronic lymphocytes and macrophages with type-II pneumocyte hyperplasia. Highresolution CT chest is the imaging modality of choice, with common manifestations including ground-glass opacities or patchy nodular infiltrates, predominantly in the lower lobes (60). This should ideally be done with contrast, to rule out the presence of a pulmonary embolus. Five distinct types of radiologic abnormalities of CIP have been described, including cryptogenic organizing pneumonia (COP) like ground glass opacities, interstitial, hypersensitivity, and pneumonitis not-otherwise-specified (61).

\section{Management}

Corticosteroid therapy is the mainstay of CIP management, with $>80 \%$ of CIP patients having their pneumonitis improve or resolve with corticosteroids alone. Pulmonology assessment is often warranted in any patient with suspected CIP, to evaluate for bronchoscopy or help to rule out alternative etiologies. In this patient with grade 2 CIP, ICI was temporarily held while the patient was treated with prednisone $1 \mathrm{mg} / \mathrm{kg} / \mathrm{d}$, followed by $5 \mathrm{mg} /$ week taper over 4 weeks. Interval CT imaging at 4 weeks was notable for improvement in radiographic findings. In this case, since CIP resolved, the patient's ICI-therapy was restarted. While grade 1 or 2 CIP can be managed with low-dose corticosteroids and close observation, higher-grade CIP should be treated with high-dose corticosteroids (methylprednisolone IV 1-2 $\mathrm{mg} / \mathrm{kg} / \mathrm{d}$ ). Infectious disease should be consulted in addition to the pulmonary team to rule out common or unusual infections, and in some cases, empiric antimicrobials may be given when infection cannot be completely excluded. Lung biopsies are typically not warranted, but may be useful rule out infection or lymphangitic tumor spread. Patients that do not demonstrate clinical improvement in CIP within $48-72 \mathrm{~h}$ should be considered for second-line therapies, options include infliximab, mycophenolate mofetil, IVIG, or cyclophosphamide. Retrospective studies have noted that up to $86 \%$ of CIP improves with corticosteroid treatment, however, of concern there was very poor response to additional immunosuppression $(8,62)$. In addition, a recent retrospective analysis has found that pneumonitis is associated with worse survival (63). There is 
currently a deficit in evidence for salvage treatment in steroid refractory patients. This is an area under current development to ameliorate the morbidity and mortality associated with respiratory-induced adverse events.

\section{ENDOCRINE}

\section{Case 1: Hypophysitis}

Clinical presentation: A 70-year old male with advanced renal cell carcinoma receiving Ipilimumab/Nivolumab, presents with new-onset fatigue and dizziness after 2 cycles of therapy.

Immune-related endocrine events pose a clinical challenge as symptoms are often subtle. Patients can present with non-specific symptoms including nausea, vomiting, dizziness, headache, fatigue, and malaise. The pituitary, thyroid, pancreas, and adrenal glands are the organs most commonly affected, although parathyroid involvement has also been reported (64). The incidence of immune-related endocrinopathies was approximately $10 \%$ in a recent meta-analysis of 7,551 patients that received ICI (63). The risk of endocrine irAE is greatest with combination therapy, with rates of hypothyroidism (17\%), hypophysitis $(13 \%)$, and hyperthyroidism $(10 \%)$ reported $(4,65)$.

\section{Diagnostic Evaluation}

In this particular patient, a physical examination was notable for intact visual fields, however, laboratory assessment showed mild hyponatremia, with both low TSH and free T4. Morning ACTH and cortisol were also low. MRI brain was notable for pituitary enhancement.

In patients with suspected hypophysitis, the pituitaryhypothalamic axis should be examined including free T4, TSH, LH, FSH, ACTH, and cortisol, as well as serum electrolytes. It is imperative to discern between primary vs. secondary hormonal deficiencies, as this will guide appropriate management. Clinicians should recognize that hypophysitis can result in secondary adrenal insufficiency and hypothyroidism. Failure to recognize this disease entity can have negative consequences for patient care; replacing thyroid hormone prior to cortisol repletion can precipitate adrenal crisis.

\section{Management}

Ipilimumab/Nivolumab therapy was temporarily held. In consultation with endocrinology, the patient was started on hydrocortisone $10 / 5 \mathrm{mg}$ in morning and afternoon, respectively. One week later he was started on a weight-based dose of levothyroxine. The patient was provided with "sick day" instructions for stress dosing of hydrocortisone and a medical alert bracelet. The patient demonstrated clinical improvement and was restarted on PD-1 monotherapy.

It is recommended that ICI is held for any-grade hypophysitis. Once patients demonstrate stability on hormonal replacement, ICI can be restarted. Higher-grade hypophysitis (grade 3+) can be managed with an initial pulsed dose of corticosteroids. Free T4 should be monitored for levothyroxine replacement. Key concepts of management include high index of clinical suspicion, appropriate localization of endocrine dysfunction, replacement of hormones and close monitoring. Immune-related endocrine events are unique as the manifestations are often irreversible and patients often require lifelong hormone replacement (66).

\section{Case 2: Hypothyroidism}

Clinical presentation: A 60 year old female patient with stageIII lung adenocarcinoma treated with durvalumab, has a thyroid stimulating hormone (TSH) of $8.5 \mathrm{mIU} / \mathrm{l}$ with normal free thyroxine (fT4). She was asymptomatic.

Hypothyroidism is one of the most common irAEs from anti-PD-1, anti-PD-L1, and anti-CTLA-4 ICIs. A systematic review and meta-analysis by Barroso-Sousa et al. demonstrated that the overall incidence of hypothyroidism was $6.6 \%$ (65). Hypothyroidism can present with fatigue, unintentional weight gain, cold intolerance, constipation, myalgia, and dry skin.

\section{Diagnostic Evaluation}

Physical examination may be notable for goiter, bradycardia, diastolic hypertension, or delayed deep tendon reflexes. TSH and fT4 should be completed prior to initiation of ICI therapy and should be monitored every 4-6 weeks. It is important to differentiate primary from secondary hypothyroidism as discussed above, as well as differentiate hypothyroidism from late-phase thyroiditis. Elevated TSH with low fT4 is indicative of biochemical hypothyroidism. Upon detection, thyroid peroxidase (TPO) antibody should also be sent.

\section{Management}

Durvalumab therapy was continued. At 4 week follow-up, TSH level was noted to be elevated to $12 \mathrm{mIU} / \mathrm{ml}$ with normal fT4. She remained asymptomatic. However, given TSH $>10 \mathrm{mIU} / \mathrm{l}$, she was commenced on $75 \mathrm{mcg}$ of levothyroxine daily. In patients with grade 1 hypothyroidism, ICIs may be continued with close monitoring of TSH and fT4. For grade 2 toxicity, appropriate thyroid supplementation should be administered with either continued ICIs or temporary withholding until symptomatic patients with any level of TSH elevation or in asymptomatic patients with TSH levels that persist $>10 \mathrm{mIU} / \mathrm{l}$ (measured 4 weeks apart) improve. Grade 3 and 4 toxicities should be treated as grade 2 unless signs of myxedema (decreased mental status, hypotension, hypoglycemia, bradycardia, hypothermia) are present, in which case hospitalization for supportive therapy may be recommended. In general, TSH should be monitored every 6-8 weeks while titrating hormone replacement until a normal TSH is reached, with repeat testing annually or as clinically indicated.

\section{HEMATOLOGY}

\section{Case 1: Thrombocytopenia}

Clinical presentation: A 50-year old female with PD-L1+ metastatic lung adenocarcinoma presents with petechiae after 3 cycles of pembrolizumab treatment.

Hematologic irAE that may occur from anti-PD-1/PDL1/CTLA-4 include autoimmune hemolytic anemia, acquired thrombotic thrombocytopenia, hemolytic uremic syndrome, immune mediated thrombocytopenia, lymphopenia, and acquired hemophilia. Thrombocytopenia due to ICI is 
relatively infrequent, with reports ranging from 1 to $28 \%$ of patients (67-69).

\section{Diagnostic Evaluation}

In patients who develop thrombocytopenia during ICI therapy, other etiologies for this presentation should be considered, including bone marrow suppression, infiltration, platelet destruction, or platelet sequestration, with a differential diagnosis of myelodysplastic syndrome, disseminated intravascular coagulation, ICI-mediated thrombocytopenia, acquired thrombotic thrombocytopenia (TTP), and hemolytic uremic syndrome (HUS). A thorough history is important to evaluate for drug/toxin exposures or viral infections that may have led to thrombocytopenia. In this patient, $\mathrm{CBC}$ was notable for normal hemoglobin with grade 2 thrombocytopenia (platelets $70,000 / \mu \mathrm{l}$ ). Renal function was normal. There was no evidence of platelet consumption or hemolysis on a peripheral blood smear. Hemolysis labs including serum lactate dehydrogenase (LDH), haptoglobin, indirect bilirubin, and $\mathrm{CBC}$ were normal. HIV, hepatitis $\mathrm{B} / \mathrm{C}$ virus and $H$. pylori were negative. Thus, ICI-mediated immune thrombocytopenia was the most likely diagnosis.

\section{Management}

In this patient, ICI was held for 2 weeks, and a repeat $\mathrm{CBC}$ did not show improvement in platelet count until prednisone $1 \mathrm{mg} / \mathrm{kg} /$ dose was started. Re-evaluation at 2 weeks revealed improvement to grade 1 thrombocytopenia (Platelets 90,000/ $\mu \mathrm{l}$ ). Prednisone was tapered over 4 weeks, and the patient was able to be recommenced on pembrolizumab.

Most patients with low-grade thrombocytopenia improve with ICI withholding and initiation of oral corticosteroids. For higher-grade toxicities, a hematology service should be consulted for consideration of additional therapies for severe toxicity, such as IVIG, rituximab, cyclosporine A, mycophenolate mofetil, cyclophosphamide, or thrombopoietin receptor agonists. If indicated, IVIG initial dosing is recommended at $1 \mathrm{~g} / \mathrm{kg}$ as a one-time dose which can be repeated if necessary (70).

\section{GASTROINTESTINAL}

\section{Case 1: Colitis}

Clinical presentation: A 45-year old male with advanced melanoma presents to the ED with abdominal pain, non-bloody diarrhea ( $>7$ bowel movements per day) and fever 2 days after receiving 3 rd dose of combination Ipilimumab/Nivolumab therapy.

The most common GI toxicities reported from anti-CTLA-4 ICIs are diarrhea and colitis. Colitis is defined as inflammation of the lining of the colon, as opposed to diarrhea that is defined as increased number of bowel movements.

The incidence of colitis ranges from 8 to $27 \%$ with rates of diarrhea reported up to $54 \%$ of CTLA- 4 treated patients. The incidence is greatest in patients treated with CTLA-4 monotherapy vs. CTLA-4/PD-1 combination therapy $(71,72)$. When PD-1 inhibitors were compared directly with CTLA4 inhibitors, the relative risk of all-grade diarrhea and colitis was 0.58 and 0.16 , respectively (73). Combination PD-1/CTLA4 related deaths were recently found to be most frequently from colitis, accounting for $37 \%$ of fatalities (14). The time of onset is typically 5-10 weeks following initiation of therapy (74). ICI-induced hepatotoxicity has also been well-described, with incidence of $2-10 \%$ of patients receiving monotherapy (75) and $25-30 \%$ of patients being treated with combination PD1/CTLA-4 therapy (75). Hepatitis has been found to be the second most common irAE leading to fatal outcomes anti-PD1/PD-L1 therapy (14). The time of onset of hepatitis may also occur early in a patient's treatment course; typically commencing within the first 6-12 weeks after treatment initiation (76). Other less frequently reported GI toxicities include dysphagia, gastritis, duodenitis, and pancreatitis (77).

\section{Diagnostic Evaluation}

Diagnostic work-up for colitis includes standard laboratory testing to assess for infectious vs. non-infectious etiologies including CBC, CMP, ESR, and CRP. Stool cultures (bacterial, viral, ova, and parasites) should be obtained as well as stool calprotectin or lactoferrin to monitor disease activity, extrapolated from the management of inflammatory bowel disease. In patients with severe colitis, tuberculosis testing should be completed in potential preparation for the use of anti-TNF$\alpha$ inhibitors for steroid-refractory colitis. Patients should also undergo radiologic imaging with a $\mathrm{CT}$ abdomen, which may show mesenteric vessel engorgement, colonic wall thickening, colonic distension, and pericolonic fat stranding (76). In severe cases, in those in which the diagnosis is uncertain, and to evaluate the severity of colitis, direct visualization of the colon with either a colonoscopy or flexible sigmoidoscopy should be considered. The presence of ulceration on direct visualization is associated with a corticosteroid-refractory course, and early infliximab can be considered in these cases. ICI-induced colitis has been reported to have greater predilection for descending colon (see Figure 2G) $(71,76)$. Endoscopy is also useful in monitoring colitis, particularly when resumption of therapy is being planned.

This patient underwent extensive evaluation as delineated above. CT imaging revealed thickening of descending colon with pericolonic fat stranding. Gastroenterology were consulted who completed colonoscopy, which demonstrated diffuse ulceration at the descending colon.

\section{Management}

The patient was managed with intravenous fluids and prednisone $1 \mathrm{mg} / \mathrm{kg} /$ day. This resulted in clinical improvement, with reduction in bowel movement to three times per day. The patient was continued on the initial corticosteroid dose for a further 4 days, and then steroids were tapered over 4 weeks. Upon completion of the prednisone taper, nivolumab monotherapy was resumed.

Patients with grade $2+$ colitis should have ICI withheld, and treatment should only be resumed if toxicity improves to $<$ grade 1. Cases of grade $3+$ colitis often imply permanent discontinuation of anti-CTLA-4 therapy (74). Early intervention with corticosteroids and best supportive care with hydration, 
electrolyte repletion and antidiarrheal agents is crucial. If patients do not demonstrate adequate clinical response within $48-72 \mathrm{~h}$ of corticosteroid therapy, TNF-blocker therapy with infliximab should be commenced. This can be repeated again at a 2 -week interval. Vedolizumab, an anti-integrin $\alpha 4 \beta 7$ antibody, should be considered in patients who develop colitis that is refractory to infliximab or in cases that anti-TNF- $\alpha$ therapy is contraindicated.

\section{DISCUSSION}

Currently, there is a paucity of literature on the precise immunopathogenesis of specific irAEs, however several mechanisms have been suggested that vary depending on the irAE in question.

A mechanism for irAE development due to enhanced $\mathrm{T}$ cell activity against shared antigens across normal and cancer cells is supported by several preclinical models in autoimmune myocarditis and autoimmune dilated cardiomyopathy $(78,79)$. In addition, abrogation of $\mathrm{PD}-1$ pathway may contribute to the pathogenesis of rheumatoid arthritis and giant cell arteritis $(80,81)$. Furthermore, vitiligo is the most common cutaneous irAE in patients with melanoma, likely as a consequence of shared antigens on melanoma cells and melanocytes (82). Elevated levels of inflammatory cytokines are also likely involved in the pathophysiology of irAE. Both PD-1 and CTLA-4 therapies have been found to promote Th1- and Th17-mediated immune responses resulting in elevated circulating levels of IL-17 and IFN- $\gamma$ (83-85). Insights into the pathogenesis of ipilimumabinduced enterocolitis has facilitated the use of steroid-sparing agents such as infliximab and vedolizumab in this particular $\operatorname{irAE}(86,87)$. Another potential mechanism is that of increased levels of pre-existing autoantibodies seen with ICI-induced myasthenia gravis, autoimmune hemolytic anemia, autoimmune thyroiditis and type 1 diabetes mellitus (88-92). Finally, CTLA4 therapy has been found to cause hypophysitis, likely due to enhanced complement-mediated inflammation due to direct binding of anti-CTLA-4 antibody with CTLA-4 expressed on normal pituitary tissue (93). Further insights would allow the development of personalized and targeted therapies for patients, potentially reducing exposure to prolonged immunosuppression, thereby enhancing efficacy and reducing toxicity of treatment.

The association of irAE with efficacy of ICI therapy is also under investigation. As detailed previously, development of vitiligo has demonstrated a significant association with beneficial clinical outcomes (39). A recent multicenter retrospective study of patients with advanced NSCLC treated with nivolumab monotherapy found that the development of irAEs was a strong predictor of survival outcomes (94). Indeed, patients who experienced two or more irAEs had a more pronounced survival benefit, suggesting a mechanistic association between irAEs and immunotherapy efficacy. These findings have not been universally confirmed, but certainly raises the question of whether the degree of immune activation is associated with superior treatment effect.

Risk factors for the development of irAEs are also being evaluated. The clinical manifestations, timing, and severity of irAE appear to depend on immunotherapy regimen, with increased frequency and severity demonstrated with combination therapies (4, 5, 65). Patients with pre-exiting autoimmune disease appear to be at increased risk of irAE. A number of retrospective studies have indicated that while patients with pre-existing may be at increased risk for both exacerbation of their autoimmune condition and for de novo immune-related adverse, this should not preclude treatment (95). Studies regarding other risk factors for developing irAE are lacking. There is likely a genetic predisposition to irAE given the role of genetics in autoimmune disease, indeed, in PD-1 deficient mice, genetic backgrounds were found to have different degree and divergent autoimmune conditions (79, 96, 97). Cappelli et al. recently evaluated immunogenetics in patients with ICIinduced IA and found that patients of European descent were more likely to be positive for HLA-DRB1 shared epitope alleles than population controls (98). Furthermore, patients with CTLA4 gene variant $1661 \mathrm{~A}>\mathrm{G}$ may predispose melanoma patients to the development of endocrine irAEs (99). In addition, the JHH group have recently demonstrated that in NSCLC, tumor histologic type may be a risk factor for CIP development and that the development of CIP worsens survival in patients receiving immunotherapy $(58,59)$.

A new wave of research is focused on identification of irAE biomarkers, which would enable identification of populations at higher risk for development of irAE. Both obesity and smoking result in pro-inflammatory state (100102). An "inflamed phenotype" may exist that could affect both therapeutic responses and toxicity risk. It may also be of benefit to account for the baseline inflammatory profiles present in the patient prior to ICI, but this is an area that requires additional research. The risk of both therapeutic and adverse effects of ICI may also be modulated by the gut microbiota (103). Preclinical studies have found that Bifidobacteria promote the natural antitumor immune responses, possibly by inducing a favorable shift toward Th1 responses (104). Patients treated with CTLA-4 therapy with a predominance of bacteria from the Bacteroidetes phylum were found to have reduced rates of ipilimumab-induced colitis (105). These findings raise the possibility that variations in gastrointestinal flora that affect host immunity influence the risk of irAE. Indeed, differences in the intestinal microflora of patients may explain some of the heterogeneity in immunotherapeutic and toxicity outcomes in patients receiving ICI therapy. A number of therapeutic avenues are currently being explored. Anderson and Rapport recently suggested a biomarker profile incorporating IL-17/IFN- $\gamma / \mathrm{IL}-$ $10 /$ TGF- $\beta 1 /$ CRP based on findings from ipilimumab-associated colitis (106). Other potential biomarkers include measurement of circulating neutrophil activation using myeloperoxidase/matrix metalloproteinase 9/L-selectin (107).

\section{CONCLUSION}

Immunotherapy is associated with a unique side effect profile that can result in significant morbidity and mortality. Immunerelated toxicities require prompt recognition and intervention to optimize clinical outcomes. As immunotherapy enters common clinical practice, it is crucial that multidisciplinary collaborations are established to improve the recognition and management of common or serious irAE, in particular. In this emerging field, 
an anti-cancer therapy that is able to selectively kill cancer cells without causing toxicities to normal cells, remains elusive. Further research is required to enhance our understanding and to define the immunopathogenesis of irAE so that strategies for prevention, early detection and targeted therapy can be developed.

\section{CONSENT}

Written and informed institutional consent was provided for the use of clinical images. The clinical cases presented in this review are fictional.

\section{REFERENCES}

1. Hanahan D, Weinberg, R. Hallmarks of cancer: the next generation. Cell. (2011) 144:646-74. doi: 10.1016/j.cell.2011.02.013

2. Eggermont A, Chiarion-Sileni V, Grob J, Dummer R, Wolchok J, Schmidt $\mathrm{H}$, et al. Prolonged survival in stage III melanoma with ipilimumab adjuvant therapy. N Engl J Med. (2016) 375:1845-55. doi: 10.1056/NEJMoa1611299

3. Hodi F, O’Day S, McDermott D, Weber R, Sosman J, Haanen J, et al. Improved survival with ipilimumab in patients with metastatic melanoma. N Engl J Med. (2010) 363:711-23. doi: 10.1056/NEJMoa1003466

4. Larkin J, Chiarion-Sileni V, Gonzalez R, Grob J, Cowey C, Lao C, et al. Combined nivolumab and ipilimumab or monotherapy in untreated melanoma. N Engl J Med. (2015) 373:23-34. doi: 10.1056/NEJMoa1504030

5. Hellmann M, Ciuleanu T, Pluzanski A, Lee J, Otterson G, AudigierValette C, et al. Nivolumab plus ipilimumab in lung cancer with a high tumor mutational burden. N Engl J Med. (2018) 378:2093-104. doi: 10.1056/NEJMoa1801946

6. Hammers H, Plimack E, Infante J, Rini B, McDermott D, Lewis L, et al. Safety and efficacy of nivolumab in combination with ipilimumab in metastatic renal cell carcinoma: the CheckMate 016 study. J Clin Oncol. (2017) 35:3851-8. doi: 10.1200/JCO.2016.72.1985

7. Abdel-Rahman O, ElHalawani H, Fouad M. Risk of endocrine complications in cancer patients treated with immune check point inhibitors: a metaanalysis. Future Oncol. (2016) 12:413-25. doi: 10.2217/fon.15.222

8. Naidoo J, Wang X, Woo K, Iyriboz T, Halpenny D, Cunningham J, et al. Pneumonitis in patients treated with anti-programmed death1/programmed death ligand 1 therapy. J Clin Oncol. (2017) 35:709-17. doi: 10.1200/JCO.2016.68.2005

9. Morganstein D, Lai Z, Spain L, Diem S, Levine D, Mace C, et al. Thyroid abnormalities following the use of cytotoxic T-lymphocyte antigen4 and programmed death receptor protein-1 inhibitors in the treatment of melanoma. Clin Endocrinol. (2017) 86:614-20. doi: 10.1111/cen.13297

10. Brahmer J, Lacchetti C, Thompson J. Management of immune-related adverse events in patients treated with immune checkpoint inhibitor therapy: American Society of clinical oncology clinical practice guideline summary. $J$ Oncol Pract. (2018) 14:247-9. doi: 10.1200/JOP.18.00005

11. Nccn.org. (2019). Available online at: https://www.nccn.org/professionals/ physician_gls/pdf/immunotherapy.pdf (accessed February 25, 2019).

12. Haanen J, Carbonnel F, Robert C, Kerr K, Peters S, Larkin J, Jordan K. Management of toxicities from immunotherapy: ESMO clinical practice guidelines for diagnosis, treatment and follow-up. Ann Oncol. (2018) 29(Suppl. 4):iv264-iv266. doi: 10.1093/annonc/mdy162

13. Puzanov I, Diab A, Abdallah K, Bingham C, Brogdon C, Dadu R, et al. Managing toxicities associated with immune checkpoint inhibitors: consensus recommendations from the Society for Immunotherapy of Cancer (SITC) Toxicity Management Working Group. J Immunother Cancer. (2017) 5:95. doi: 10.1186/s40425-017-0300-Z

14. Wang D, Salem J, Cohen J, Chandra S, Menzer C, Ye F, et al. Fatal toxic effects associated with immune checkpoint inhibitors. JAMA Oncol. (2018) 4:1721-8. doi: 10.1001/jamaoncol.2018.3923

\section{AUTHOR CONTRIBUTIONS}

This review was drafted by $\mathrm{CC}$ and $\mathrm{KB}$ and critically revised by JN.

\section{ACKNOWLEDGMENTS}

We would like to acknowledge the contributions of members of the JHH irAE Toxicology Board who provided clinical images including Dr. Tawnie Braaten, Dr. Laura Cappelli, Dr. Roberta Florido, Dr. Shawn Kwatra, and Dr. John Probasco.

15. Santini F, Rizvi H, Plodkowski A, Ni A, Lacouture M, GambarinGelwan M, et al. Safety and efficacy of re-treating with immunotherapy after immune-related adverse events in patients with NSCLC. Cancer Immunol Res. (2018) 6:1093-9. doi: 10.1158/2326-6066.CIR17-0755

16. Lidar M, Giat E, Garelick D, Horowitz Y, Amital H, SteinbergSilman Y, et al. Rheumatic manifestations among cancer patients treated with immune checkpoint inhibitors. Autoimmun Rev. (2018) 17:284-9. doi: 10.1016/j.autrev.2018.01.003

17. Calabrese L, Calabrese C, Cappelli L. Rheumatic immune-related adverse events from cancer immunotherapy. Nat Rev Rheumatol. (2018) 14:569-79. doi: 10.1038/s41584-018-0074-9

18. Cappelli L, Brahmer J, Forde P, Le D, Lipson E, Naidoo J, et al. Clinical presentation of immune checkpoint inhibitor-induced inflammatory arthritis differs by immunotherapy regimen. Semin Arthritis Rheum. (2018) 48:553-7. doi: 10.1016/j.semarthrit.2018.02.011

19. Cappelli L, Gutierrez A, Baer A, Albayda J, Manno R, Haque $\mathrm{U}$, et al. Inflammatory arthritis and sicca syndrome induced by nivolumab and ipilimumab. Ann Rheum Dis. (2016) 76:43-50. doi: 10.1136/annrheumdis-2016-209595

20. Calabrese C, Kirchner E, Kontzias K, Velcheti V, Calabrese L. Rheumatic immune-related adverse events of checkpoint therapy for cancer: case series of a new nosological entity. RMD Open. (2017) 3:e000412. doi: 10.1136/rmdopen-2016-000412

21. Buder-Bakhaya K, Benesova K, Schulz C, Anwar H, DimitrakopoulouStrauss A, Weber $\mathrm{T}$, et al. Characterization of arthralgia induced by PD-1 antibody treatment in patients with metastasized cutaneous malignancies. Cancer Immunol Immunother. (2017) 67:175-82. doi: 10.1007/s00262-017-2069-9

22. Le Burel S, Champiat S, Mateus C, Marabelle A, Michot J, Robert $\mathrm{C}$, et al. Prevalence of immune-related systemic adverse events in patients treated with anti-programmed cell death 1/anti-programmed cell death-ligand 1 agents: a single-centre pharmacovigilance database analysis. Eur J Cancer. (2017) 82:34-44. doi: 10.1016/j.ejca.2017. 05.032

23. Smith M, Bass A. Arthritis after cancer immunotherapy: symptom duration and treatment response. Arthritis Care Res. (2018) 71:362-6. doi: $10.1002 /$ acr. 23467

24. Salem J, Manouchehri A, Moey M, Lebrun-Vignes B, Bastarache L, Pariente A, et al. Cardiovascular toxicities associated with immune checkpoint inhibitors: an observational, retrospective, pharmacovigilance study. Lancet Oncol. (2018) 19:1579-89. doi: 10.1016/S1470-2045(18) 30608-9

25. Proven A, Gabriel S, Orces C, O'Fallon W, Hunder G. Glucocorticoid therapy in giant cell arteritis: duration and adverse outcomes. Arthritis Rheum. (2003) 49:703-8. doi: 10.1002/art.11388

26. Langford C, Cuthbertson D, Ytterberg S, Khalidi N, Monach P, Carette $\mathrm{S}$, et al. A randomized, double-blind trial of abatacept (CTLA-4Ig) for the treatment of takayasu arteritis. Arthritis Rheumatol. (2017) 69:846-53. doi: 10.1002/art.40037 
27. Varricchi G, Galdiero M, Marone G, Criscuolo G, Triassi M, Bonaduce D, et al. Cardiotoxicity of immune checkpoint inhibitors. ESMO Open. (2017) 2:e000247. doi: 10.1136/esmoopen-2017-000247

28. Johnson DB, Balko JM, Compton ML, Chalkias S, Gorham J, Xu Y, et al. Fulminant myocarditis with combination immune checkpoint blockade. $N$ Engl J Med. (2016) 375:1749-55. doi: 10.1056/NEJMoa1609214

29. Mahmood S, Fradley M, Cohen J, Nohria A, Reynolds K, Heinzerling L, et al. Myocarditis in patients treated with immune checkpoint inhibitors. $\mathrm{J} \mathrm{Am}$ Coll Cardiol. (2018) 71:A699. doi: 10.1016/j.jacc.2018.02.037

30. Dec G, Palacios I, Fallon J, Aretz H, Mills J, Lee D, et al. Active myocarditis in the spectrum of acute dilated cardiomyopathies. N Engl J Med. (1985) 312:885-90. doi: 10.1056/NEJM198504043121404

31. Escudier M, Cautela J, Malissen N, Ancedy Y, Orabona M, Pinto $\mathrm{J}$, et al. Clinical features, management, and outcomes of immune checkpoint inhibitor-related cardiotoxicity. Circulation. (2017) 136:2085-7. doi: 10.1161/CIRCULATIONAHA.117.030571

32. Lyon A, Yousaf N, Battisti N, Moslehi J, Larkin J. Immune checkpoint inhibitors and cardiovascular toxicity. Lancet Oncol. (2018) 19:e447-e458. doi: 10.1016/S1470-2045(18)30457-1

33. Villadolid J, Amin A. Immune checkpoint inhibitors in clinical practice: update on management of immune-related toxicities. Transl Lung Cancer Res. (2015) 4:560-75. doi: 10.3978/j.issn.2218-6751.2015.06.06

34. Robert C, Schachter J, Long G, Arance A, Grob J, Mortier L, et al. Pembrolizumab versus ipilimumab in advanced melanoma. $N$ Engl J Med. (2015) 372:2521-32. doi: 10.1056/NEJMoa1503093

35. Bertrand A, Kostine M, Barnetche T, Truchetet M, Schaeverbeke T. Immune related adverse events associated with anti-CTLA-4 antibodies: systematic review and meta-analysis. BMC Med. (2015) 13:211. doi: 10.1186/s12916-015-0455-8

36. Baum S, Sakka N, Artsi O, Trau H, Barzilai A. Diagnosis and classification of autoimmune blistering diseases. Autoimmun Rev. (2014) 13:482-9. doi: 10.1016/j.autrev.2014.01.047

37. Freeman-Keller M, Kim Y, Cronin H, Richards A, Gibney G, Weber J. Nivolumab in resected and unresectable metastatic melanoma: characteristics of immune-related adverse events and association with outcomes. Clin Cancer Res. (2015) 22:886-94. doi: 10.1158/1078-0432.CCR-15-1136

38. Hua C, Boussemart L, Mateus C, Routier E, Boutros C, Cazenave H, et al. Association of vitiligo with tumor response in patients with metastatic melanoma treated with pembrolizumab. JAMA Dermatol. (2016) 152:45. doi: 10.1001/jamadermatol.2015.2707

39. Teulings H, Limpens J, Jansen S, Zwinderman A, Reitsma J, Spuls $\mathrm{P}$, et al. Vitiligo-like depigmentation in patients with stage III-IV melanoma receiving immunotherapy and its association with survival: a systematic review and meta-analysis. J Clin Oncol. (2015) 33:773-81. doi: 10.1200/JCO.2014.57.4756

40. Gandhi L, Rodríguez-Abreu D, Gadgeel S, Esteban E, Felip E, De Angelis F, et al. Pembrolizumab plus chemotherapy in metastatic non-small-cell lung cancer. N Engl J Med. (2018) 378:2078-92. doi: 10.1056/NEJMoal 801005

41. Cortazar F, Marrone K, Troxell M, Ralto K, Hoenig M, Brahmer J, et al. Clinicopathological features of acute kidney injury associated with immune checkpoint inhibitors. Kidney Int. (2016) 90:638-47. doi: 10.1016/j.kint.2016.04.008

42. Shirali A, Perazella M, Gettinger S. Association of acute interstitial nephritis with programmed cell death 1 inhibitor therapy in lung cancer patients. Am J Kidney Dis. (2016) 68:287-91. doi: 10.1053/j.ajkd.2016.02.057

43. Weber J, D'Angelo S, Minor D, Hodi F, Gutzmer R, Neyns B, et al. Nivolumab versus chemotherapy in patients with advanced melanoma who progressed after anti-CTLA-4 treatment (CheckMate 037): a randomised, controlled, open-label, phase 3 trial. Lancet Oncol. (2015) 16:375-84. doi: 10.1016/S1470-2045(15)70076-8

44. Topalian S, Sznol M, McDermott D, Kluger H, Carvajal R, Sharfman W, et al. Survival, durable tumor remission, and long-term safety in patients with advanced melanoma receiving nivolumab. J Clin Oncol. (2014) 32:1020-30. doi: 10.1200/JCO.2013.53.0105

45. Sznol M, Ferrucci P, Hogg D, Atkins M, Wolter P, Guidoboni M, et al. Pooled analysis safety profile of nivolumab and ipilimumab combination therapy in patients with advanced melanoma. J Clin Oncol. (2017) 35:3815-22. doi: 10.1200/JCO.2016.72.1167

46. Wanchoo R, Karam S, Uppal N, Barta V, Deray G, Devoe C, et al. Adverse renal effects of immune checkpoint inhibitors: a narrative review. Am J Nephrol. (2017) 45:160-9. doi: 10.1159/000455014

47. Larkin J, Chmielowski B, Lao C, Hodi F, Sharfman W, Weber J, et al. Neurologic serious adverse events associated with nivolumab plus ipilimumab or nivolumab alone in advanced melanoma, including a case series of encephalitis. Oncologist. (2017) 22:709-18. doi: 10.1634/theoncologist.2016-0487

48. Cuzzubbo S, Tissier M. P04.08 Neurological adverse events associated with the immune checkpoint inhibitors: review of the literature and characterization of the neurological patterns. Neuro Oncol. (2016) 18(Suppl. 4):iv25-iv26. doi: 10.1093/neuonc/now188.086

49. Fellner A, Makranz C, Lotem M, Bokstein F, Taliansky A, Rosenberg S, et al. Neurologic complications of immune checkpoint inhibitors. J Neuro Oncol. (2018) 137:601-9. doi: 10.1007/s11060-018-2752-5

50. Liao B, Shroff S, Kamiya-Matsuoka C, Tummala S. A typical neurological complications of ipilimumab therapy in patients with metastatic melanoma. Neuro Oncol. (2014) 16:589-93. doi: 10.1093/neuonc/nou001

51. Johnson D, Saranga-Perry V, Lavin P, Burnette W, Clark S, Uskavitch $\mathrm{D}$, et al. Myasthenia gravis induced by ipilimumab in patients with metastatic melanoma. J Clin Oncol. (2015) 33:e122-e124. doi: 10.1200/jco.2015.33.15_suppl.9019

52. Suzuki S, Ishikawa N, Konoeda F, Seki N, Fukushima S, Takahashi K, et al. Nivolumab-related myasthenia gravis with myositis and myocarditis in Japan. Neurology. (2017) 89:1127-34. doi: 10.1212/WNL.0000000000004359

53. Makarious D, Horwood K, Coward J. Myasthenia gravis: an emerging toxicity of immune checkpoint inhibitors. Eur J Cancer. (2017) 82:128-36. doi: 10.1016/j.ejca.2017.05.041

54. Abdel-Rahman O, Oweira H, Petrausch U, Helbling D, Schmidt J, Mannhart $\mathrm{M}$, et al. Immune-related ocular toxicities in solid tumor patients treated with immune checkpoint inhibitors: a systematic review. Expert Rev Anticancer Ther. (2017) 17:387-94. doi: 10.1080/14737140.2017.1296765

55. Dalvin L, Shields C, Orloff M, Sato T, Shields J. Checkpoint inhibitor immune therapy. Retina. (2018) 38:1063-78. doi: 10.1097/IAE.0000000000002181

56. Rosenbaum J, Pasadhika S. Update on the use of systemic biologic agents in the treatment of noninfectious uveitis. Biologics. (2014) 8:67-81. doi: 10.2147/BTT.S41477

57. Rashdan S, Minna J, Gerber D. Diagnosis and management of pulmonary toxicity associated with cancer immunotherapy. Lancet Respir Med. (2018) 6:472-8. doi: 10.1016/S2213-2600(18)30172-3

58. Suresh K, Voong K, Shankar B, Forde P, Ettinger D, Marrone K, et al. Pneumonitis in non-small cell lung cancer patients receiving immune checkpoint immunotherapy: incidence and risk factors. J Thorac Oncol. (2018) 13:1930-9. doi: 10.1016/j.jtho.2018.08.2035

59. Suresh K, Psoter K, Voong K, Shankar B, Forde P, Ettinger D, et al. Impact of checkpoint inhibitor pneumonitis on survival in NSCLC patients receiving immune checkpoint immunotherapy. J Thorac Oncol. (2019) 14:494-502. doi: 10.1016/j.jtho.2018.11.016

60. Voong K, Hazell S, Hu C, Hayman J, Hales R, Marrone K, et al. MA 09.08 receipt of chest radiation and immune-related pneumonitis in patients with NSCLC treated with anti-PD-1/PD-L1. J Thorac Oncol. (2017) 12:S1837. doi: 10.1016/j.jtho.2017.09.529

61. O'Kane G, Labbé C, Doherty M, Young K, Albaba H, Leighl N. Monitoring and management of immune-related adverse events associated with programmed cell death protein-1 axis inhibitors in lung cancer. Oncologist. (2016) 22:70-80. doi: 10.1634/theoncologist.2016-0164

62. Naidoo J, Page D, Li B, Connell L, Schindler K, Lacouture M, et al. Toxicities of the anti-PD-1 and anti-PD-L1 immune checkpoint antibodies. Ann Oncol. (2016) 27:1362. doi: 10.1093/annonc/mdw141

63. El Majzoub I, Qdaisat A, Thein K, Win M, Han M, Jacobson K, et al. Adverse effects of immune checkpoint therapy in cancer patients visiting the emergency department of a comprehensive cancer center. Ann Emerg Med. (2019) 73:79-87. doi: 10.1016/j.annemergmed.2018.04.019

64. Callahan M, Wolchok J. At the bedside: CTLA-4- and PD-1-blocking antibodies in cancer immunotherapy. J Leuk Biol. (2013) 94:41-53. doi: $10.1189 /$ jlb.1212631 
65. Barroso-Sousa R, Barry W, Garrido-Castro A, Hodi F, Min L, Krop I, et al. Incidence of endocrine dysfunction following the use of different immune checkpoint inhibitor regimens. JAMA Oncol. (2018) 4:173. doi: 10.1001/jamaoncol.2017.3064

66. Hodi F, Chesney J, Pavlick A, Robert C, Grossmann K, McDermott D, et al. Combined nivolumab and ipilimumab versus ipilimumab alone in patients with advanced melanoma: 2-year overall survival outcomes in a multicentre, randomised, controlled, phase 2 trial. Lancet Oncol. (2016) 17:1558-68. doi: 10.1016/S1470-2045(16)30366-7

67. Weber J, Postow M, Lao C, Schadendorf D. Management of adverse events following treatment with anti-programmed death-1 agents. Oncologist. (2016) 21:1230-40. doi: 10.1634/theoncologist.2016-0055

68. Shiuan E, Beckermann K, Ozgun A, Kelly C, McKean M, McQuade $\mathrm{J}$, et al. Thrombocytopenia in patients with melanoma receiving immune checkpoint inhibitor therapy. J Immunother Cancer. (2017) 5:8. doi: 10.1186/s40425-017-0210-0

69. Sharma P, Callahan M, Bono P, Kim J, Spiliopoulou P, Calvo E, et al. Nivolumab monotherapy in recurrent metastatic urothelial carcinoma (CheckMate 032): a multicentre, open-label, two-stage, multi-arm, phase 1/2 trial. Lancet Oncol. (2016) 17:1590-8. doi: 10.1016/S1470-2045(16)30496-X

70. Neunert C, Lim W, Crowther M, Cohen A, Solberg L, Crowther M. The American Society of Hematology 2011 evidence-based practice guideline for immune thrombocytopenia. Blood. (2011) 117:4190-207. doi: 10.1182/blood-2010-08-302984

71. Gupta A, De Felice K, Loftus E, Khanna S. Systematic review: colitis associated with anti-CTLA-4 therapy. Aliment Pharmacol Ther. (2015) 42:406-17. doi: 10.1111/apt.13281

72. Cabanillas G. Immune related adverse events and their treatment in melanoma patients receiving ipilimumab. J Clin Oncol. (2017) 35(15 suppl):e14598. doi: 10.1200/JCO.2017.35.15_suppl.e14598

73. Bishay K, Tandon P, Bourassa-Blanchette S, Parlow S, Laurie S, McCurdy J. 453 - the risk of diarrhea and colitis in patients with lung cancer undergoing immune-checkpoint inhibitor therapy. Gastroenterology. (2018) 154:S-104S-105. doi: 10.1016/S0016-5085(18)30787-X

74. Marthey L, Mateus C, Mussini C, Nachury M, Nancey S, Grange F, et al. Cancer immunotherapy with anti-CTLA-4 monoclonal antibodies induces an inflammatory bowel disease. J Crohn Colitis. (2016) 10:395-401. doi: 10.1093/ecco-jcc/jjv227

75. Ziemer M, Koukoulioti E, Beyer S, Simon J, Berg T. Managing immune checkpoint-inhibitor-induced severe autoimmune-like hepatitis by liver-directed topical steroids. J Hepatol. (2017) 66:657-9. doi: 10.1016/j.jhep.2016.11.015

76. Cramer P, Bresalier R. Gastrointestinal and hepatic complications of immune checkpoint inhibitors. Curr Gastroenterol Rep. (2017) 19:3. doi: $10.1007 /$ s1 1894-017-0540-6

77. Abdel-Rahman O, ElHalawani H, Fouad M. Risk of gastrointestinal complications in cancer patients treated with immune checkpoint inhibitors: a meta-analysis. Immunotherapy. (2015) 7:1213-27. doi: 10.2217/imt.15.87

78. Nishimura H. Autoimmune dilated cardiomyopathy in PD-1 receptordeficient mice. Science. (2001) 291:319-22. doi: 10.1126/science.291.5502.319

79. Okazaki T, Tanaka Y, Nishio R, Mitsuiye T, Mizoguchi A, Wang J, et al. Autoantibodies against cardiac troponin I are responsible for dilated cardiomyopathy in PD-1-deficient mice. Nat Med. (2003) 9:1477-83. doi: $10.1038 / \mathrm{nm} 955$

80. Guo Y, Walsh A, Canavan M, Wechalekar M, Cole S, Yin X, et al. Immune checkpoint inhibitor PD-1 pathway is down-regulated in synovium at various stages of rheumatoid arthritis disease progression. PLoS ONE. (2018) 13:e0192704. doi: 10.1371/journal.pone.0192704

81. Zhang H, Watanabe R, Berry G, Vaglio A, Liao Y, Warrington $\mathrm{K}$, et al. Immunoinhibitory checkpoint deficiency in medium and large vessel vasculitis. Proc Natl Acad Sci USA. (2017) 114:E970-E979. doi: 10.1073/pnas.1616848114

82. Larsabal M, Marti A, Jacquemin C, Rambert J, Thiolat D, Dousset L, et al. Vitiligo-like lesions occurring in patients receiving anti-programmed cell death -1 therapies are clinically and biologically distinct from vitiligo. J Am Acad Dermatol. (2017) 76:863-70. doi: 10.1016/j.jaad.2016.10.044

83. Bamias G, Delladetsima I, Perdiki M, Siakavellas S, Goukos D, Papatheodoridis G, et al. Immunological characteristics of colitis associated with anti-CTLA-4 antibody therapy. Cancer Investig. (2017) 35:443-55. doi: 10.1080/07357907.2017.1324032

84. Callahan M, Yang A, Tandon S, Xu Y, Subudhi S, Roman R, et al. Evaluation of serum IL-17 levels during ipilimumab therapy: correlation with colitis. J Clin Oncol. (2011) 29(15 Suppl):2505. doi: 10.1200/jco.2011.29.15_suppl.2505

85. Dulos J, Carven G, van Boxtel S, Evers S, Driessen-Engels L, Hobo W, et al. PD-1 Blockade augments Th1 and Th17 and suppresses Th2 responses in peripheral blood from patients with prostate and advanced melanoma cancer. J Immunother. (2012) 35:169-78. doi: 10.1097/CJI.0b013e318247a4e7

86. Yanai S, Nakamura S, Matsumoto T. Nivolumab-induced colitis treated by infliximab. Clin Gastroenterol Hepatol. (2017) 15:e80-e81. doi: 10.1016/j.cgh.2016.09.017

87. Bergqvist V, Hertervig E, Gedeon P, Kopljar M, Griph H, Kinhult $\mathrm{S}$, et al. Vedolizumab treatment for immune checkpoint inhibitorinduced enterocolitis. Cancer Immunol Immunother. (2017) 66:581-92. doi: 10.1007/s00262-017-1962-6

88. Shirai T, Sano T, Kamijo F, Saito N, Miyake T, Kodaira M, et al. Acetylcholine receptor binding antibody-associated myasthenia gravis and rhabdomyolysis induced by nivolumab in a patient with melanoma. Jpn J Clin Oncol. (2015) 46:86-8. doi: 10.1093/jjco/hyv158

89. Kobayashi T, Iwama S, Yasuda Y, Okada N, Tsunekawa T, Onoue T, et al. Patients with antithyroid antibodies are prone to develop destructive thyroiditis by nivolumab: a prospective study. J Endocr Soc. (2018) 2:241-51. doi: 10.1210/js.2017-00432

90. Kong B, Micklethwaite K, Swaminathan S, Kefford R, Carlino M. Autoimmune hemolytic anemia induced by anti-PD-1 therapy in metastatic melanoma. Melanoma Res. (2016) 26:202-4. doi: 10.1097/CMR.0000000000000232

91. Palla A, Kennedy D, Mosharraf H, Doll D. Autoimmune hemolytic anemia as a complication of nivolumab therapy. Case Rep Oncol. (2016) 9:691-7. doi: 10.1159/000452296

92. Mizokami-Stout K, Gianchandani R, Maceachern M, Iyengar R, Yentz S, Shen L, et al. A systematic review of cases of diabetes mellitus following immune checkpoint inhibitor therapy for cancer. Diabetes. (2018) 67(Suppl. 1):204-LB. doi: $10.2337 / \mathrm{db} 18-204-\mathrm{LB}$

93. Iwama S, De Remigis A, Callahan M, Slovin S, Wolchok J, Caturegli P. Pituitary expression of CTLA-4 mediates hypophysitis secondary to administration of CTLA-4 blocking antibody. Sci Transl Med. (2014) 6:230ra45. doi: 10.1126/scitranslmed.3008002

94. Ricciuti B, Genova C, De Giglio A, Bassanelli M, Dal Bello M, Metro G, et al. Impact of immune-related adverse events on survival in patients with advanced non-small cell lung cancer treated with nivolumab: long-term outcomes from a multi-institutional analysis. J Cancer Res Clin Oncol. (2018) 145:479-85. doi: 10.1007/s00432-018-2805-3

95. Johnson D, Sullivan R, Menzies A. Immune checkpoint inhibitors in challenging populations. Cancer. (2017) 123:1904-11. doi: $10.1002 / \mathrm{cncr} .30642$

96. Wang J, Yoshida T, Nakaki F, Hiai H, Okazaki T, Honjo T. Establishment of NOD-Pdcd1-/- mice as an efficient animal model of type I diabetes. Proc Natl Acad Sci USA. (2005) 102:11823-28. doi: 10.1073/pnas.0505497102

97. Nishimura H, Nose M, Hiai H, Minato N, Honjo T. Development of lupus-like autoimmune diseases by disruption of the PD-1 gene encoding an ITIM motif-carrying immunoreceptor. Immunity. (1999) 11:141-51. doi: 10.1016/S1074-7613(00)80089-8

98. Cappelli L, Dorak M, Bettinotti M, Bingham C, Shah A. Association of HLA-DRB1 shared epitope alleles and immune checkpoint inhibitorinduced inflammatory arthritis. Rheumatology. (2018) 58:476-80. doi: 10.1093/rheumatology/key358

99. Queirolo P, Dozin B, Morabito A, Banelli B, Carosio R, Fontana V, et al. CTLA-4 gene variant $-1661 \mathrm{~A}>\mathrm{G}$ may predict the onset of endocrine adverse events in metastatic melanoma patients treated with ipilimumab. Eur J Cancer. (2018) 97:59-61. doi: 10.1016/j.ejca.2018.04.005

100. Mirsoian A, Bouchlaka M, Sckisel G, Chen M, Pai C, Maverakis E, et al. Adiposity induces lethal cytokine storm after systemic administration of stimulatory immunotherapy regimens in aged mice. J Exp Med. (2014) 211:2373-83. doi: 10.1084/jem.20140116 
101. Huang C, Wang C, Fu C, Huang C, Chang P, Chen Y, et al. Association between cigarette smoking and interleukin-17A expression in nasal tissues of patients with chronic rhinosinusitis and asthma. Medicine. (2016) 95:e5432. doi: 10.1097/MD.0000000000005432

102. van Eeden S, Hogg J. The response of human bone marrow to chronic cigarette smoking. Eur Respir J. (2000) 15:915-21. doi: 10.1034/j.1399-3003.2000.15e18.x

103. Zitvogel L, Ayyoub M, Routy B, Kroemer G. Microbiome and anticancer immunosurveillance. Cell. (2016) 165:276-87. doi: 10.1016/j.cell.2016.03.001

104. Vetizou M, Pitt J, Daillere R, Lepage P, Waldschmitt N, Flament C, et al. Anticancer immunotherapy by CTLA-4 blockade relies on the gut microbiota. Science. (2015) 350:1079-84. doi: 10.1126/science.aad1329

105. Dubin K, Callahan M, Ren B, Khanin R, Viale A, Ling L, et al. Intestinal microbiome analyses identify melanoma patients at risk for checkpoint-blockade-induced colitis. Nat Commun. (2016) 7:10391. doi: 10.1038/ncomms10391

106. Anderson R, Rapoport B. Immune dysregulation in cancer patients undergoing immune checkpoint inhibitor treatment and potential predictive strategies for future clinical practice. Front Oncol. (2018) 8:80. doi: $10.3389 /$ fonc. 2018.00080

107. Shahabi V, Berman D, Chasalow S, Wang L, Tsuchihashi Z, Hu B, et al. Gene expression profiling of whole blood in ipilimumab-treated patients for identification of potential biomarkers of immune-related gastrointestinal adverse events. J Transl Med. (2013) 11:75. doi: 10.1186/1479-5876-11-75

Conflict of Interest Statement: The authors declare that the research was conducted in the absence of any commercial or financial relationships that could be construed as a potential conflict of interest.

Copyright (c) 2019 Connolly, Bambhania and Naidoo. This is an open-access article distributed under the terms of the Creative Commons Attribution License (CC BY). The use, distribution or reproduction in other forums is permitted, provided the original author(s) and the copyright owner(s) are credited and that the original publication in this journal is cited, in accordance with accepted academic practice. No use, distribution or reproduction is permitted which does not comply with these terms. 